\title{
Assessment of torso deformities using 3D markerless asymmetry analysis and its clinical applications
}

\author{
Amin Komeili', Lindsey Westover, Eric Parent, Marc Moreau, Marwan El-Rich, Samer Adeeb \\ From 10th International Conference on Conservative Management of Spinal Deformities - SOSORT 2013 \\ Annual Meeting \\ Chicago, IL, USA. 8-11 May 2013
}

\section{Background}

The location of the scoliosis curve apex is important in brace prescription and can only be obtained from radiographs [1]. Surface Topography (ST) has poor correlation with curve shape but current ST analyses often rely on markers placed by operators, which can affect measurement accuracy.

\section{Purpose}

This study aimed to meet the following objectives: (1) develop an approach to analyzing markerless torso ST data, (2) propose a ST classification system for torso asymmetry in adolescent idiopathic scoliosis (AIS) patients, and (3) predict the location of the curve apex based on ST.

\section{Methods}

The full torso ST of 90 AIS patients with different curve types were retrieved from our previous study [2]. The mean Cobb angle was $32.5^{\circ}$ (range: $8^{\circ}-69^{\circ}$ ). The best plane of symmetry that divides the torso into left and right parts was calculated. Deviations between the left and right parts were measured and displayed as deviation colour maps (DCMs). To propose a surface clarification, the DCMs of 46 patients were appraised by three scoliosis professionals. The DCMs were then classified into three main groups and six subgroups by four novice observers. The intra and inter-observer reliability of the classification was assessed using Kappa coefficients. The vertical position of the maximum deviation point above the PSISs was multiplied by a correction factor to estimate the vertical location of the curve apex.

* Correspondence: akomeili@ualberta.ca University of Alberta, Alberta, Canada

\section{Results}

The mean kappa coefficient for intra-observer reliability was $0.85(0.68-0.92)$ indicating good to excellent classification reliability [3]. The inter-observer kappa coefficient was 0.62 and the percentage of agreement was $80 \%$, indicating moderate reliability [3]. For 88 torsos with thoracic curves (subgroups 2, 4 or 5), the location of the point of maximum deviation predicted the location of the curve apex with a $\pm 2.2 \mathrm{~cm}$ accuracy (range $0.02-5.5 \mathrm{~cm}, \mathrm{R}^{2}=0.72$ ). For 39 lumbar curves with Cobb angle $>20^{\circ}$, prediction accuracy was $\pm 1.6 \mathrm{~cm}\left(0.02-4.2 \mathrm{~cm} ; R^{2}=0.42\right)$.

\section{Conclusions and discussion}

A non-invasive markerless ST quantification of the asymmetry of the torso was developed allowing reliable classification of patients with scoliosis. The new approach can predict the location of the curve apex with errors corresponding to the dimensions of one vertebra.

Published: 18 September 2013

\section{References}

1. Rigo MD, Villagrasa M, Gallo D: "A specific scoliosis classification correlating with brace treatment: description and reliability,". Scoliosis 2010, 5:1-7161-5-1.

2. Parent EC, Zhang PQ, Hill D, Moreau M, Hedden D, Lou E: "Sensitivity-tochange of Full Torso Surface Topography Measurements in Adolescents with Idiopathic Scoliosis and a Main Thoracic Curve,". Stud. Health Technol. Inform. 2012, 176:484.

3. Altman DG: Practical Statistics for Medical Research. London; New York: Chapman and Hall; 1991.

doi:10.1186/1748-7161-8-S2-O25

Cite this article as: Komeili et al:: Assessment of torso deformities using 3D markerless asymmetry analysis and its clinical applications. Scoliosis 2013 8(Suppl 2):025. 